\title{
Forward Error Correction Codes in Communication Channels
}

\author{
Yong-Geol Shim \\ School of Electronics and Electrical Engineering, Dankook University, \\ 152, Jukjeon-ro, Suji-gu, Yongin-si, \\ Gyeonggi-do, 16890, Korea \\ ygshim@dankook.ac.kr
}

\begin{abstract}
In this paper, forward error correction codes in communication channels are designed and proposed. The proposed codes facilitate self-synchronization of the digital communication systems. By utilizing a systematic development process, forward error correcting codes with limited the run length of zero is obtained. The filtering operations pass the complex envelope of the received signal. To facilitate the synchronization, the proposed codes possess good error correcting performance and self-synchronization capability. The codes give good performance in a wide range of adjustable complexity, and outperforms Viterbi decoding at comparable complexity. A joint regression analysis was conducted to compute the index of code rate. The codes can be applied to various digital communication systems.
\end{abstract}

Keywords: Forward Error Correction, Channel coding, Error correcting codes, communication engineering, electronics

\section{Introduction}

In communication systems, it is required to limit the run length of zero to facilitate the synchronization. [1] The addition of error correcting codes is indispensable to maintain the reliability of the systems. For radio frequency communication, relatively sloppy analog filters are used in the field and at intermediate frequencies for super-heterodyne reception. The complex version of these filtering operations corresponds to passing the complex envelope of the received signal through a sloppy analog complex filter, which is a cascade of the complex baseband versions of the analog filters used in the receive chain.

The error correcting codes have the property which facilitate self-synchronization. The codes can efficiently operate and improve the performance of digital communication systems. Multicarrier modulation, transforms a system with memory into a system in the frequency domain, by decomposing the channel into parallel narrowband channels, each of which sees a scalar channel gain. The sampling rate is chosen to be an integer multiple of the symbol rate. This provides a front end which yields a discrete signal [2-3].

We provide an introduction to some commonly used channel coding techniques. The key idea of channel coding is to introduce redundancy in the transmitted signal so as to enable recovery from channel impairments such as errors and erasures. We know from the previous chapter that, for any given set of channel conditions, there exists a Shannon capacity, or maximum rate of reliable transmission. Such Shannon-theoretic limits provide the ultimate benchmark for channel code design.

A large number of error control techniques are available to the modern communication system designer, and in this paper, we provide a small subset of the candidate code words. Our emphasis is on block codes, which have been a workhorse of communication link design for many decades, and forward error correction codes, which have revolutionized communication systems by enabling implementable designs that approach Shannon capacity for a variety of channel models. 
The effect of fading is mitigated using multiple codes at transmitter or receiver. The sampled outputs of the analog filter can be processed directly by an adaptive digital filter that is determined by the specific equalization algorithm employed. While the matched filter is an analog filter, as discussed earlier, it can be implemented in discrete time using samples at the output of a wideband analog filter. The matched filter is implemented in discrete time after estimating the effective discrete time channel from the input to the transmit filter to the output of the sampler after the analog filter.

The wireless sensor nodes are too small to accommodate multiple antennas on a single terminal. The nodes can cooperate to obtain cooperative diversity. For the suboptimal equalization techniques that we discuss, it is not necessary to implement the matched filter.

The techniques developed in this paper apply to single-carrier systems in which data are sent using linear modulation. An alternative technique for handling dispersive channels is the use of multicarrier modulation, or orthogonal frequency division multiplexing.

\section{Forward Error Correction Codes}

A channel output provides useful information about its inputs. There are many strategies to achieve self-synchronization. [4] In forward error correcting method, the nodes retransmit amplified versions of the signal received from source. Parallel concatenation of convolutional codes is proposed in this paper. An information sequence $\mathrm{u}$ is fed into a convolutional encoder. To get good performance, it turns out that the encoder should be chosen to be recursive. We employ our recursive systematic code. The information sequence is then permuted and fed to another convolutional encoder.

The information sequence and the two parity sequences [5-7] are then modulated and sent through the channel. We use BPSK over an AWGN channel. Thus, if the constituent encoders are rate $1 / 2$, then the overall code thus obtained is of rate $1 / 3$. A higher rate can be achieved using the same construction simply by not transmitting some of the bits generated by the encoders. This procedure is referred to as puncturing. Note that a punctured block code can be decoded in the same manner as one without puncturing, by interpreting the bits not sent as erasures.

We do not consider puncturing in our discussion here. In the iterative decoding both decoders see the channel output for the information sequence. Decoder 1 sees the channel output for the parity sequence for encoder, while decoder sees the channel output for the parity sequence from encoder. The decoders exchange information about the information sequence.

We provide a method to some commonly used channel coding techniques. The key idea of channel coding is to introduce redundancy in the transmitted signal so as to enable recovery from channel impairments such as errors and erasures. [8-11] For any given set of channel conditions, there exists a Shannon capacity, or maximum rate of reliable transmission. Such Shannon-theoretic limits provide the ultimate benchmark for channel code design. Suppose, now, that we wish to estimate the parameter. The channel does not depend on the receiver device, and does not affect the MLE in both cases. In general, we can choose to define the density with respect to any convenient measure, to get a form that is easy to manipulate. This is the idea we use to define the notion of a density for a continuous time signal. We define the density as the likelihood function of a hypothesis corresponding to the model with respect to a dummy hypothesis that is independent of the signal.

The transmitted complex baseband signal is converted to pass-band using a local oscillator at carrier frequency. Both the local oscillator and the sampling clock are often integer or rational multiples of the natural frequency of a crystal oscillator, and can be generated using a phase locked loop. 
The coefficients at the nodes determine the performance at the destination. The channels provide complete information about one of the inputs. A distributed joint sourcechannel coding technique exploits source relay correlation. The throughput of can be increased further by using error correcting codes at the nodes.

The coding makes the packets to be combined at the nodes. Let (K P, F) be the probability space. Let $\mathrm{F}$ be a two-user binary-input multiple-access channel with

$$
\left|I_{(1)}\left(P_{+}\right)\right|-I_{(1)}(P)<d .
$$

The binary linear graph can be represented by a Tanner graph using a parity check matrix. [12-13] A Tanner graph is a bipartite graph with variable nodes on the left corresponding to the different code symbols, and check nodes on the right, one for each check equation, or row of the parity check matrix. A variable node is connected to a check node if it participates in the corresponding parity check equation. The Tanner graph corresponding to the parity check matrix for the $(7,4)$ Hamming code is used. The first check node corresponds to the first row of the parity check matrix. Low density parity check codes are described in terms of a parity check matrix. The parity check matrices are not unique, so it is also possible to find parity check matrices for the same code. However, the sparseness of the parity check matrix that we do use is crucial for both code construction and iterative decoding.

The framework for demodulation developed in this paper exploits signal space techniques to project the continuous-time signal to a finite-dimensional vector space, and then applied detection theory to characterize optimal receivers. We now wish to apply a similar strategy for the more general problem of parameter estimation, where the parameter may be continuous valued phase or amplitude. The resulting framework also enables us to recover, as a special case, the results derived earlier for optimal detection for signaling in AWGN, since this problem can be interpreted as that of estimating a parameter.

\section{Asymptotic Efficiency}

Consider a rectangular transmit pulse and a channel impulse response. The impulse response of the cascade of the transmit pulse and channel filter is displayed. The computation of the transfer function bound is entirely similar to the convergence analysis. The bound applies in this case as well, and the simultaneous equations relating the transfer function from the all-zero start state to all other states can be written down and solved.

However, solving for the bound as a function of transfer rate can be difficult for large iteration number. We now want to prune the terms to obtain an intelligent union bound. To do this, consider a rectangular transmit pulse and a channel impulse response. [14-16] It shows a simplified schematic channel equalization. The correct path corresponds to the all-zero error sequence.

The proposed code output makes an error in bit 27. It diverges from the correct path around bit 34, and coincides with the correct path. Instead of considering a trellis corresponding to the symbol sequence as in the development of the Viterbi algorithm, it is now convenient to consider a trellis in which a symbol sequence is represented by its error sequence relative to the transmitted sequence.

This trellis has 3 states at each time, and the transmitted sequence corresponds to the all zero path. [17] Two paths in the trellis merge when 23 successive symbols for the path are the same. Thus, a path in our error sequence trellis merges with the all-zero path corresponding to the transmitted sequence if there are 12 consecutive zeros in the error sequence. The ML sequence diverges and remerges with the transmitted sequence in several segments. [18] 
Consider now the error sequence, which coincides with the segment of the ML sequence which diverges from the true sequence around the bit of interest. Such a sequence has the property that, once it remerges with the all zero path, it never diverges again.

\section{Design Rules}

An analysis based on weight enumerator functions can also be carried out for serial concatenation of block codes. The inner code must be recursive. The forward error correcting capability is the same as twice the number of bits at the output of the outer code. The gain is given by the free distance of the outer block code.

The outer code is preferably recursive, since this leads to fewer input errors for error events at the free distance which dominate the overall code performance. This means, for example, that we could improve on the code by making the outer code the recursive version. The sampled outputs of the analog filter can be processed directly by an adaptive digital filter that is determined by the specific equalization algorithm employed. While the matched filter is an analog filter, as discussed earlier, it can be implemented in discrete time using samples at the output of a wideband analog filter.

In principle, differential modulation can also be employed with QAM alphabets, by encoding information in amplitude and phase transitions, assuming that the channel is roughly constant over several symbols, but there are technical issues with both encoding and decoding. Suppose that the complex-valued channel gains are unknown. This could occur as a result of inherent channel time variations or of imperfect carrier phase recovery. If the phase index can vary arbitrarily, then there is no hope of recovering the information encoded in the system.

Both parallel and serial concatenation of codes provide interleaving gains by decreasing the multiplicity of low weight code words in inverse proportion to the length. This is contrast to classical design, where the effort is to increase the minimum distance. While forward error correcting codes display an error floor, this can be pushed down by increasing the code length, with residual errors handled by an outer error detection code.

\section{Numerical Comparison}

In this section, we compare the performances of the decoding algorithms for binary antipodal signals. The bit error probability of the proposed code is presented. [19] The antipodal modulation is used over the additive white Gaussian noise channel. The external quantum dual code (EQDC) performance exhibits an error floor, since it does not have enough dimensions to suppress all of the inter symbol interference as the signal to noise ratio gets large. The performance is particularly poor and the linear ZF equalizer does not exist. The EQDC performs more poorly than even the linear MSE equalizer over a wide range of signal to noise ratio.

Figure 1 shows the bit error probabilities of the proposed code and priority-first search method. Figure 2 shows the calculation amount of the codes. If three or more odd number of errors occur, the decoder can detect the errors, but cannot correct them. To illustrate the performance of the equalization schemes discussed here, let us consider a numerical example for a somewhat more elaborate channel model than in our running example.

The impulse response of the cascade of the transmit pulse and channel filter is displayed. Over this channel, we transmit Gray coded QPSK symbols at a rate of 1 symbol per unit time. At the receiver front end, we use the optimal matched filter. The Tanner graph corresponding to the parity check matrix for the $(7,4)$ Hamming code is used. The first check node corresponds to the first row of the parity check matrix. Low density parity check codes are described in terms of a parity check matrix. The parity check matrices are not unique, so it is also possible to find parity check matrices for the 
same code. However, the sparseness of the parity check matrix that we do use is crucial for both code construction and iterative decoding.

It can be checked that the channel memory $\mathrm{L}=2$, so that EQDC requires 64 states. For a linear equalizer, suppose that we use an observation interval that exactly spans the impulse response for the desired symbol. It can be seen that the ZF equalizer does not exist, and that the MSE equalizer will have an error floor due to unsuppressed inter symbol interference. The ZF and EQDC can be computed. The EQDC has four feedback taps, corresponding to the four vectors. A comparison of the performance of all of the equalizers, obtained by averaging over multiple 2500 symbol packets. Note that MEF performance is almost indistinguishable from ISI-free performance. The proposed code is the best suboptimal equalizer, about $2 \mathrm{~dB}$ away from EQDC performance. The proposed code performance exhibits an error floor, since it does not have enough dimensions to suppress all of the ISI as the SNR gets large. The ZF performance is particularly poor here: the linear ZF equalizer does not exist, and the EQDC performs more poorly than even the linear MMSE equalizer over a wide range of SNR.

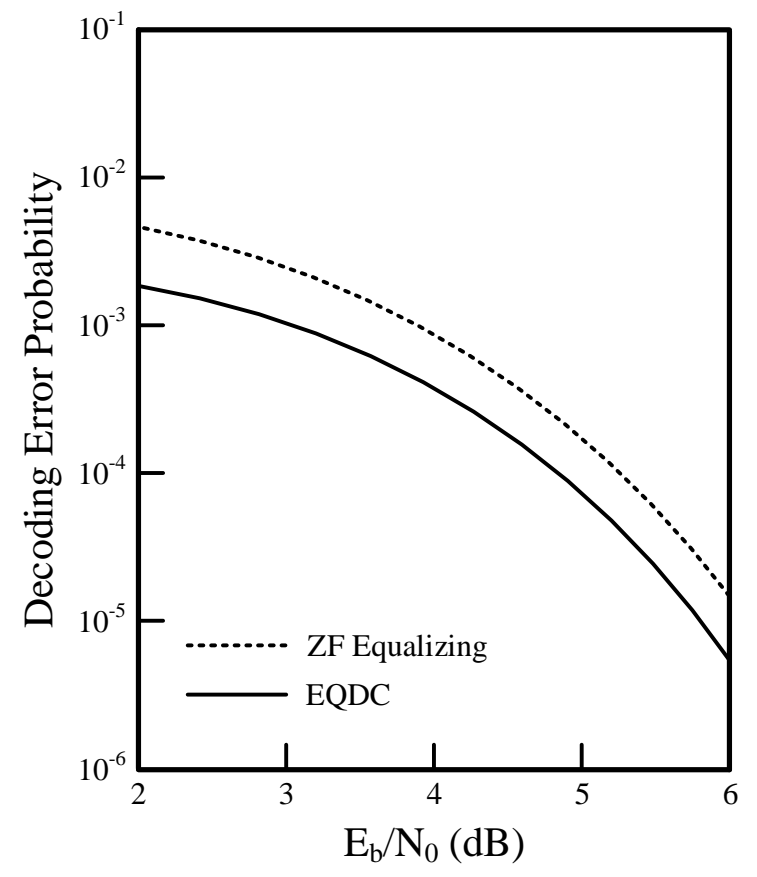

Figure 1. Decoding Error Probability of $(15,11)$ RS Code 


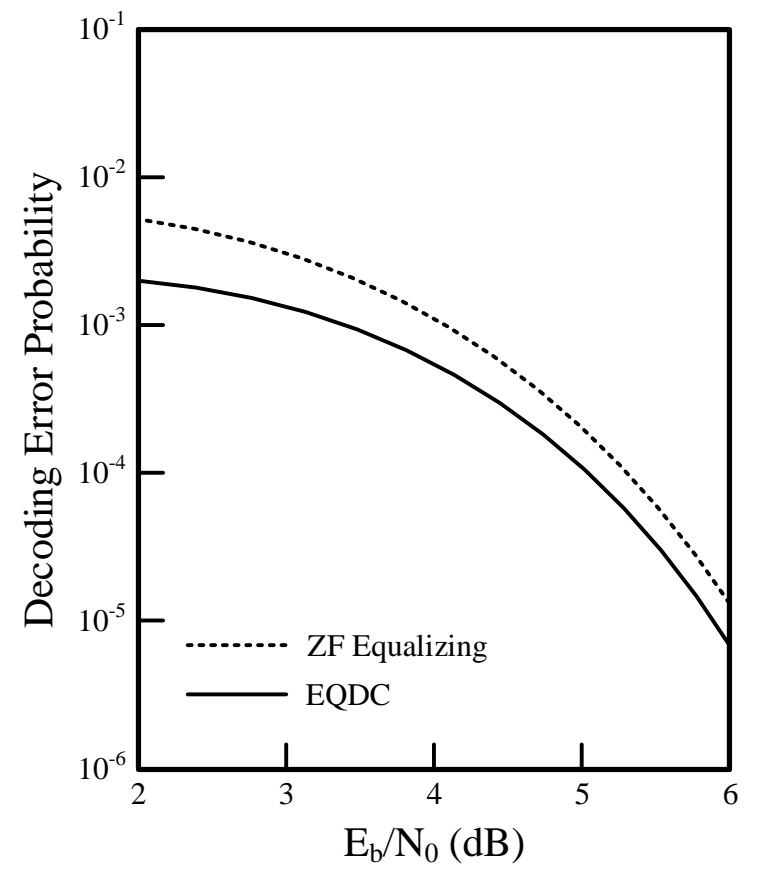

Figure 2. Decoding Error Probability of $(24,12,8)$ Extended Golay Code

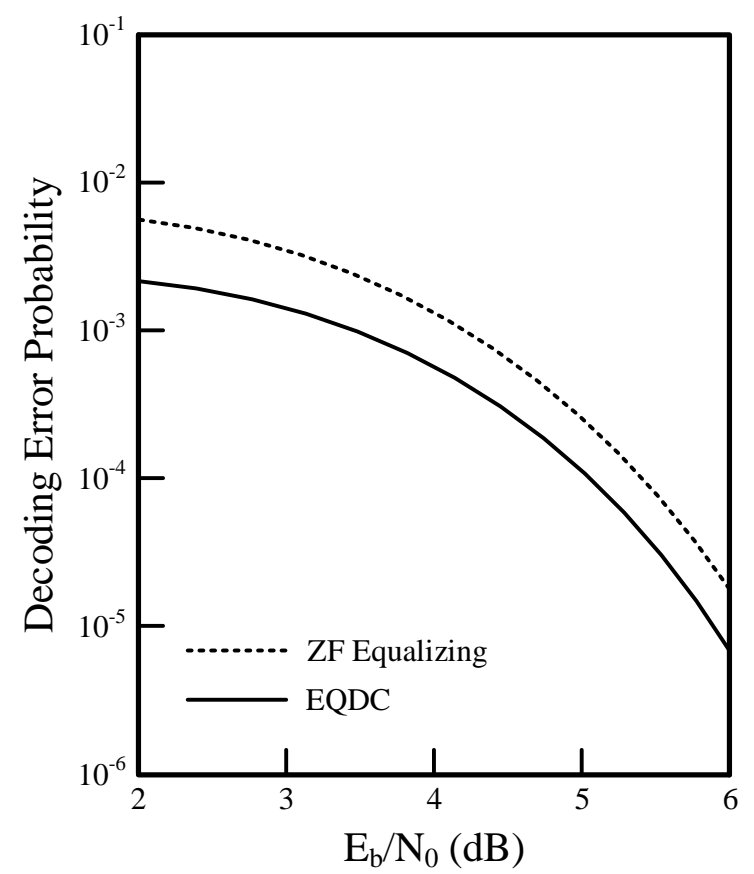

Figure 3. Decoding Error Probability of $(64,42,8)$ Reed-Muller Code 


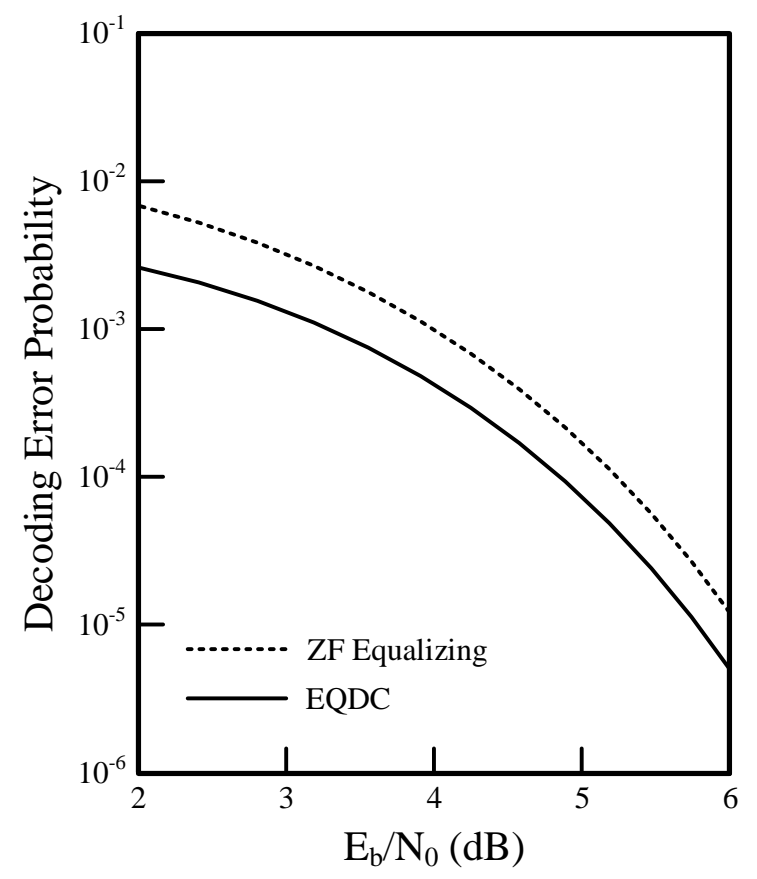

Figure 4. Decoding Error Probability of $(128,99,10)$ Extended BCH Code

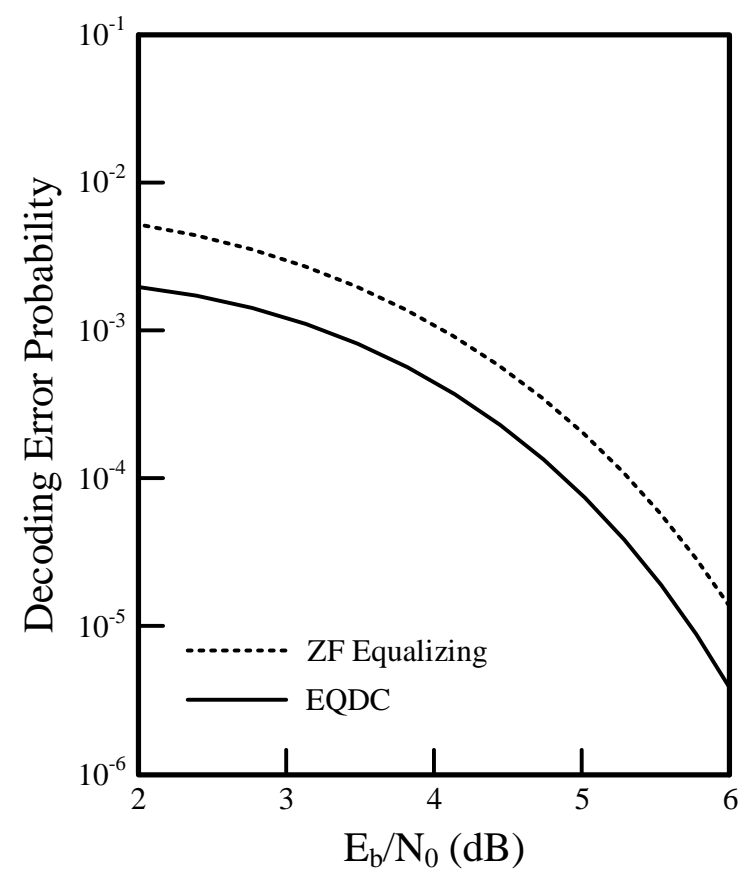

Figure 5. Decoding Error Probability of $(128,64,22)$ Extended BCH Code 


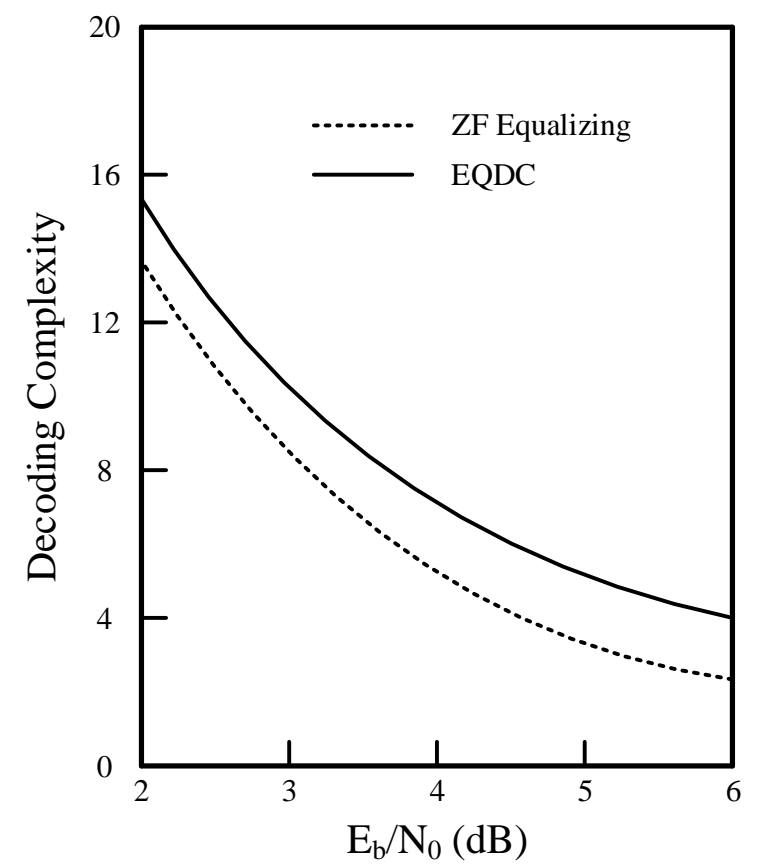

Figure 6. Decoding Complexity of $(15,11)$ RS Code

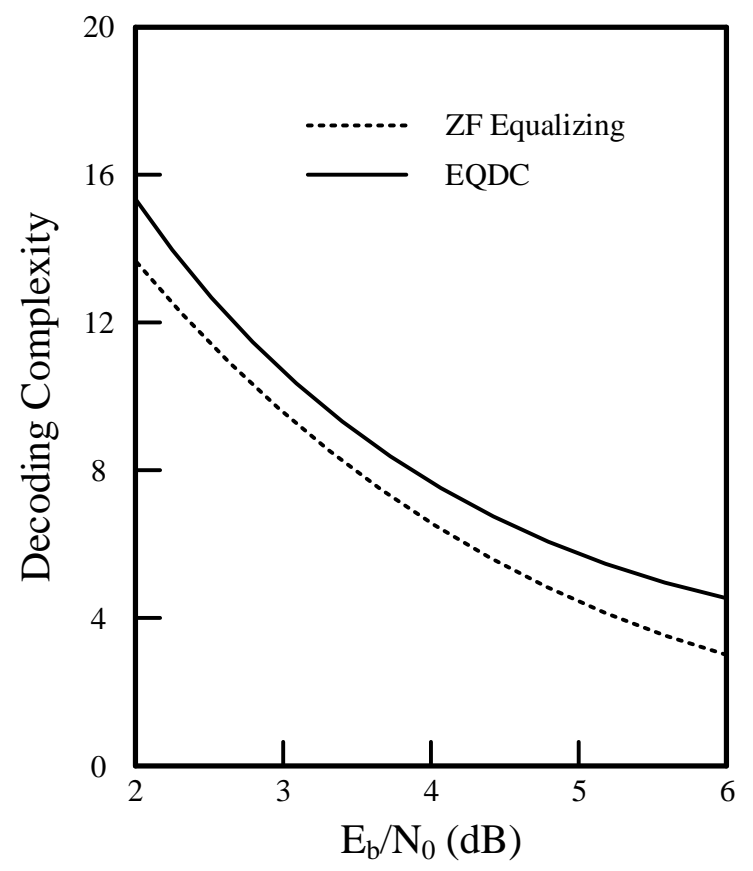

Figure 7. Decoding Complexity of $(24,12,8)$ Extended Golay Code 


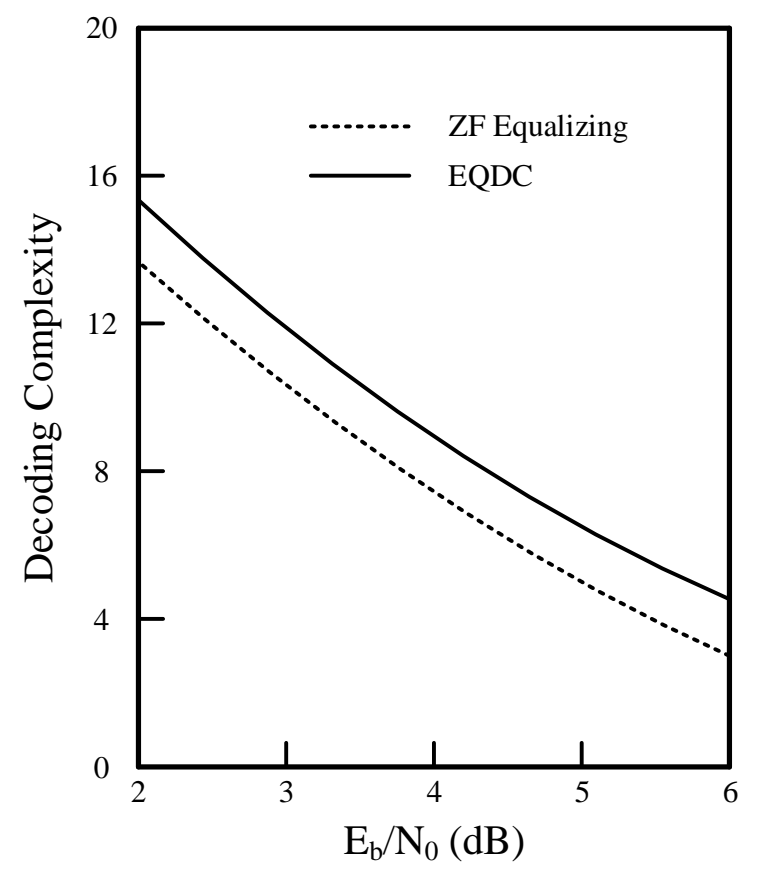

Figure 8. Decoding Complexity of $(64,42,8)$ Reed-Muller Code

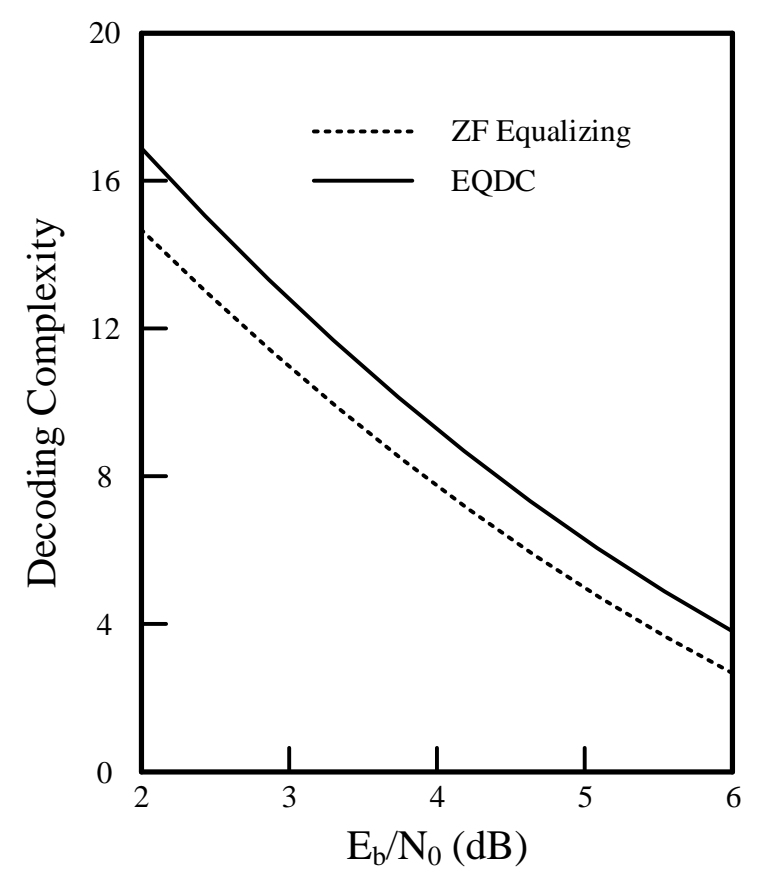

Figure 9. Decoding Complexity of $(128,99,10)$ Extended BCH Code 


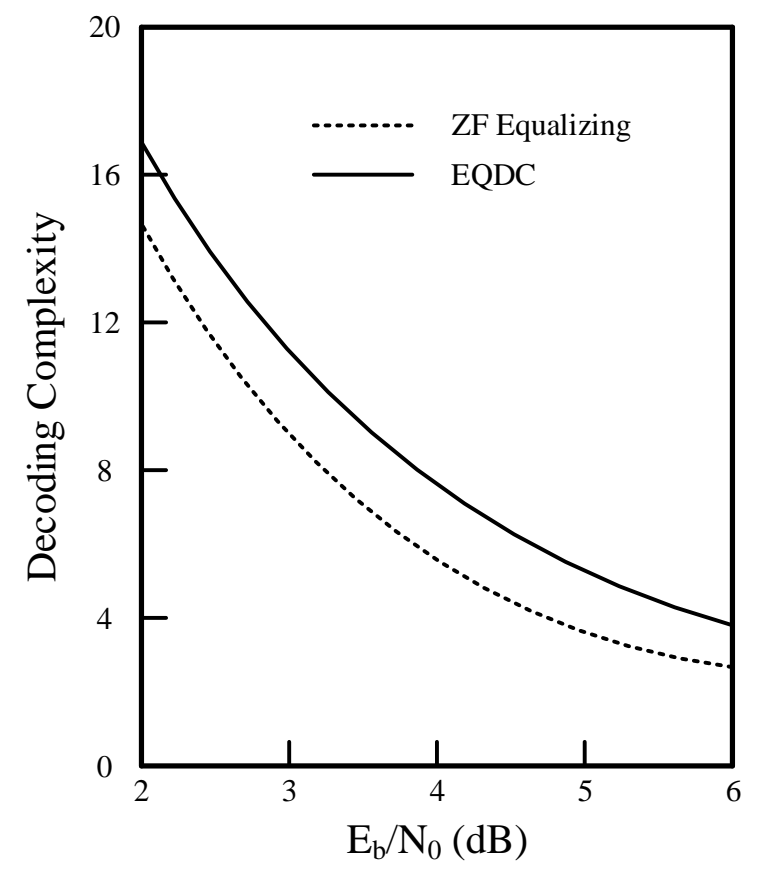

Figure 10. Decoding Complexity of $(128,64,22)$ Extended BCH Code

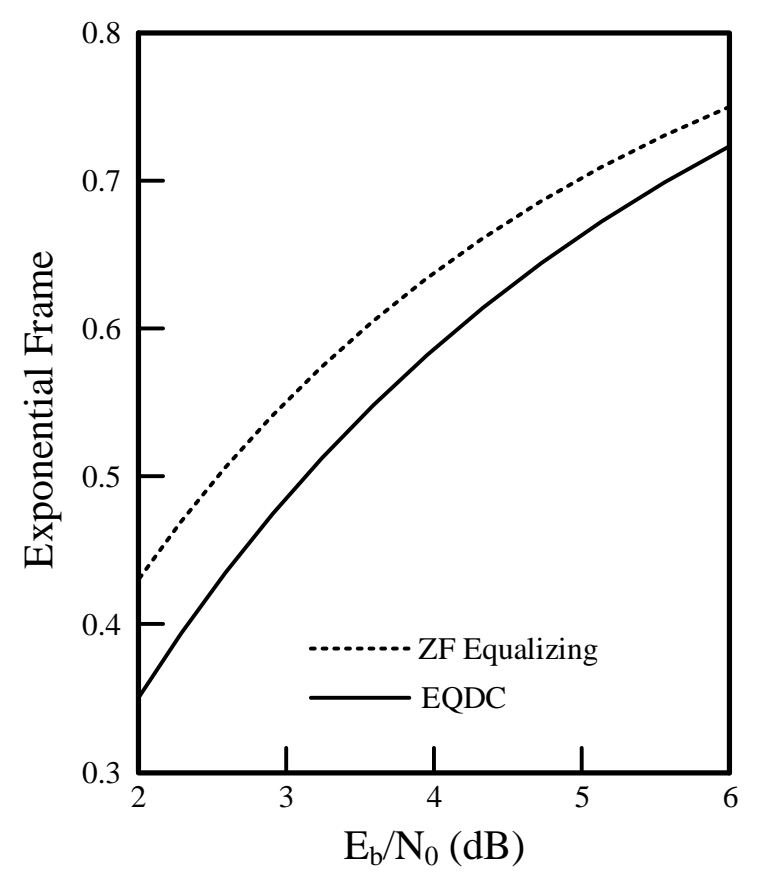

Figure 11. Exponential Frame of $(15,11)$ RS Code 


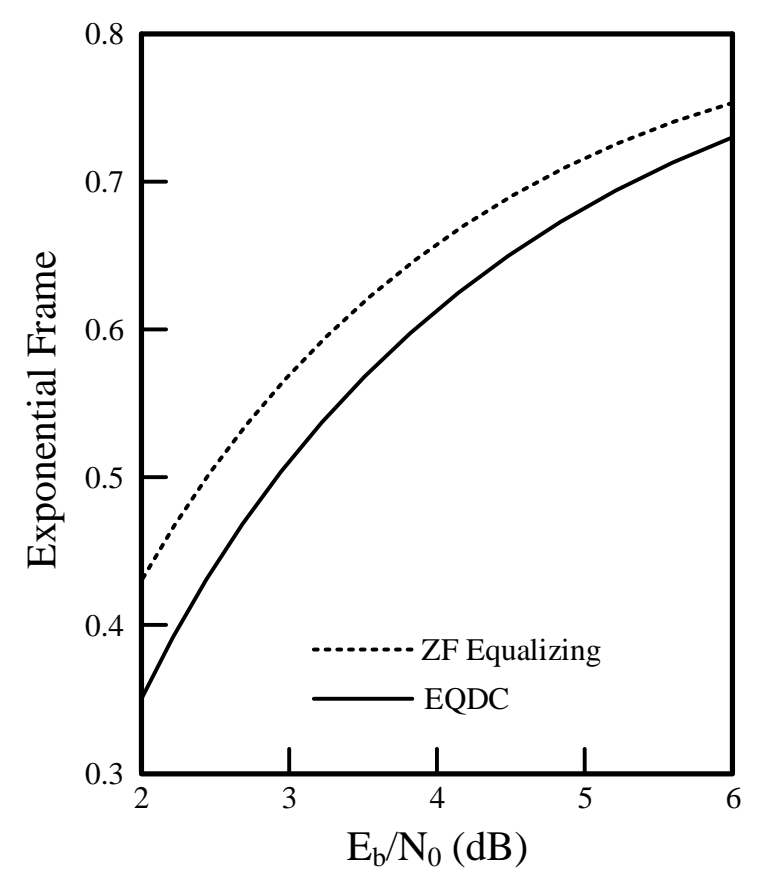

Figure 12. Exponential Frame of $(24,12,8)$ Extended Golay Code

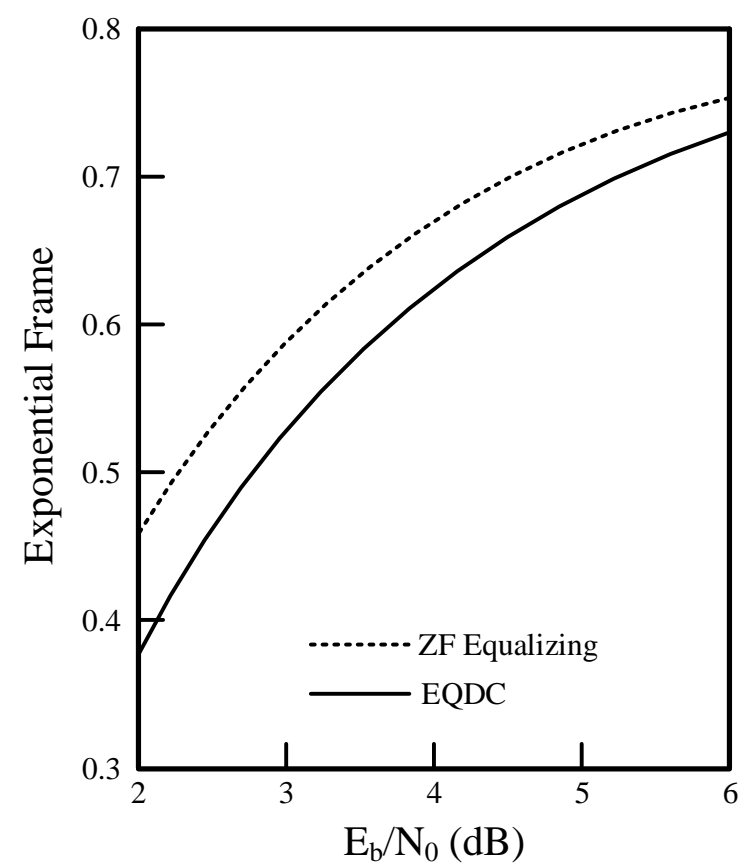

Figure 13. Exponential Frame of $(64,42,8)$ Reed-Muller Code 


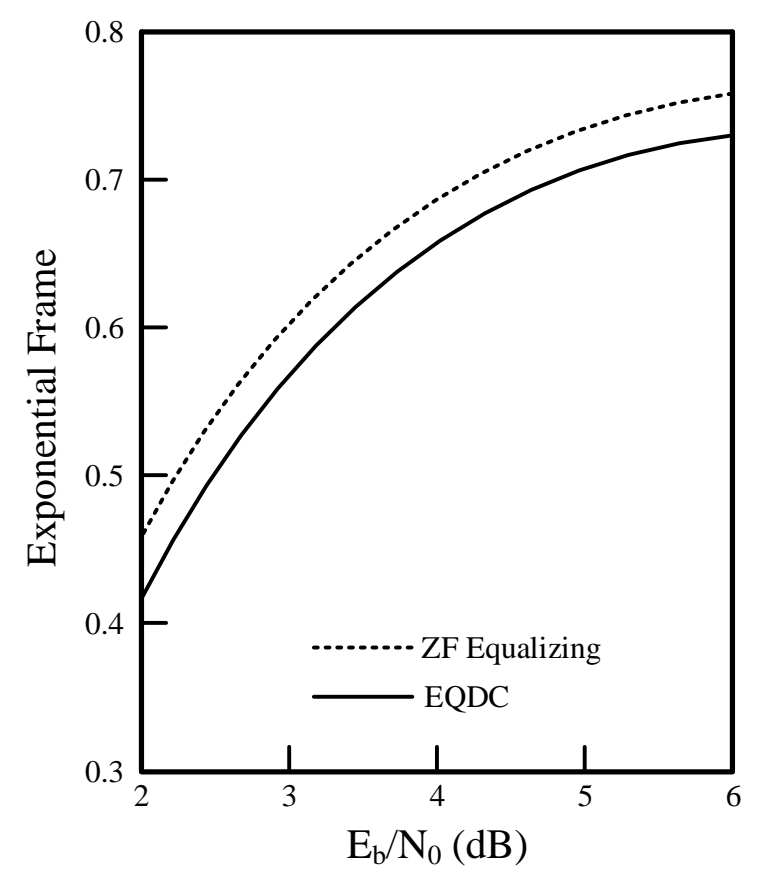

Figure 14. Exponential Frame of $(128,99,10)$ Extended BCH Code

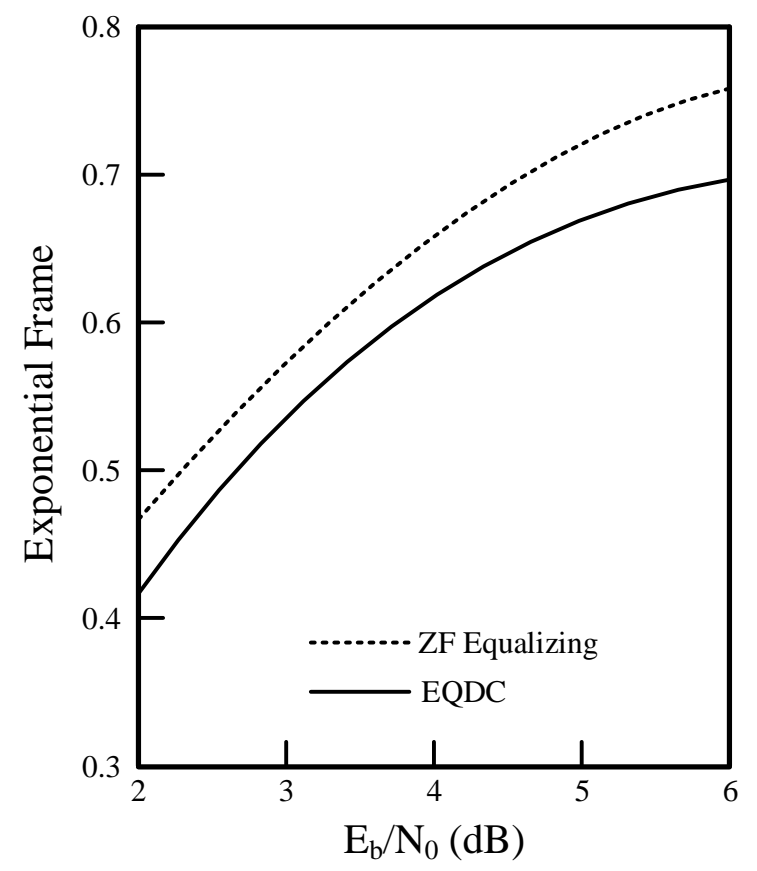

Figure 15. Exponential Frame of $(128,64,22)$ Extended BCH Code

\section{Conclusion}

An error correcting DC-Free Code for Communication Systems is designed and proposed. The proposed code facilitates self-synchronization of the digital communication systems.

The proposed code output makes an error in bit 27. It diverges from the correct path around bit 34, and coincides with the correct path. Instead of considering a trellis corresponding to the symbol sequence as in the development of the Viterbi algorithm, it is 
now convenient to consider a trellis in which a symbol sequence is represented by its error sequence relative to the transmitted sequence. To limit the run length of zero to facilitate the synchronization, it is required that the code possess good error correcting performance and self-synchronization capability. In this paper, we propose a scheme which uses BCH code for encoding. The code can be applied to various digital communication systems. The proposed code achieves self-synchronization without degradation of error correcting capability.

Both parallel and serial concatenation of codes provide interleaving gains by decreasing the multiplicity of low weight code words in inverse proportion to the length. This is contrast to classical design, where the effort is to increase the minimum distance. While forward error correcting codes display an error floor, this can be pushed down by increasing the code length, with residual errors handled by an outer error detection code. The proposed code has simple procedures for encoding and decoding. Thus it can be applied to high speed digital communication systems.

\section{Acknowledgments}

This paper is a revised and expanded version of a paper entitled "An Error Correcting DC-Free Code for Communication Systems" presented at the Third International MegaConference on Green and Smart Technology (GST 2016), Jeju Island, Korea, December 21-23, 2016.

\section{References}

[1] J. Jiang and K. R. Narayanan, "Iterative soft-input soft-output decoding of Reed-Solomon codes by adapting the parity-check matrix", IEEE Transactions on Information Theory, vol. 52, no. 8, (2006), pp. 3746-3756.

[2] G. Garrammone, "On decoding complexity of reed-solomon codes on the packet erasure channel", IEEE Communications Letters, vol. 17, no. 4, (2013), pp. 773-776.

[3] J. N. Laneman and G. W. Wornell, "Distributed space-time-coded protocols for exploiting cooperative diversity in wireless networks", IEEE Transactions on Information Theory, vol. 49, no. 10, (2003), pp. 2415-2425.

[4] J. Lee and C. C. Woo, "An Error Correcting High Rate DC-Free Multimode Code Design for Optical Storage Systems", Journal of the Institute of Signal Processing and Systems, vol. 11, no. 3, (2010) July, pp. 226-231.

[5] H. Tokushige, K. Nakamaye, T. Koumoto, Y. S. Tang and T. Kasami, "Selection of Search Centers in Iterative Soft-Decision Decoding Algorithms", IEICE Trans. on Fundamentals of Electronics, Communications and Computer Sciences, vol. E84-A, no. 10, (2001), pp. 2397-2403.

[6] Y. G. Shim, "Selection of Possible Code Words in Channel Coding Systems", International Journal of Control and Automation, vol. 6, no. 5, (2013), pp. 361-368.

[7] Y. G. Shim, "An Improvement of Soft Decision Decoding Algorithm Using Linear Block Codes", International Journal of Software Engineering and Its Applications, vol. 7, no. 6, (2013), pp. 319-324.

[8] Y. G. Shim, "Coding and Decoding Scheme Controlling Run Length", International Journal of Control and Automation, vol. 6, no. 5, (2013), pp. 315-322.

[9] W. Liying, "Design of the Distributed Framework and Dataflow Process for Urban Road Traffic Information Systems", Second International Conference on Intelligent Computation Technology and Automation, 2009. ICICTA '09, (2009), IEEE.

[10] H. Xu and M. Barth, "An adaptive dissemination mechanism for inter-vehicle communication-based decentralized traffic information systems", Intelligent Transportation Systems Conference, 2006. ITSC '06, IEEE, (2006), pp. 1207-1213.

[11] Wegener and C. Schmidt, "AutoCast: An Adaptive Data Dissemination Protocol for Traffic Information Systems”, IEEE 66th Vehicular Technology Conference, 2007. VTC-2007, IEEE Press, (2007), pp. 1947-1951.

[12] J. Ott, E. Hyytia, P. Lassila, T. Vaegs and J. Kangasharju, "Floating Content: Information Sharing in Urban Areas", 2011 IEEE International Conference on Pervasive Computing and Communications (PerCom), IEEE Press, (2011), pp. 136-146.

[13] J. K. Bulter, "Towards understanding and Measuring the Conditions of Trust: Evolution of the conditions of trust inventory", Journal of Management, vol. 17, (1991), pp. 643-663.

[14] U. Madhow, "Fundamentals of Digital Communications", Cambridge University Press, (2008).

[15] D. R. Jordan, "Transmission Systems for Communications", Bell Telephone Laboratories, (1982). 
[16] P. M. Doney and J. P. Cannon, "An Examination of the Nature of Trust in Buyer-Seller Relation- ships", Journal of Marketing, (1997), pp. 35-51.

[17] G. Lee and W. Xia, "A longitudinal experimental study on the interaction effects of persuasion quality, user training, and first-hand use on user perceptions of new information technology", Information \& Management, vol. 48, Issue 7, (2011), pp. 288-295.

[18] N. Heinze and Q. Hu, "The evolution of corporate web presence: A longitudinal study of large American companies", International Journal of Information Management, vol. 26, Issue 4, (2006), pp. 313-325. Saleem, H. Nawaz, I. Ahmed and S. M. Abbas, "Analytical Evaluation of Tri-band Printed Antenna", Information Sciences Letters, vol. 1, no. 2, (2012), pp. 85-89.

[19] Y. Shim, "An Error Correcting DC-Free Code for Communication Systems", Proceedings of the Third International Mega-Conference on Green and Smart Technology (GST 2016), Jeju Island, Korea, (2016) December 21-23.

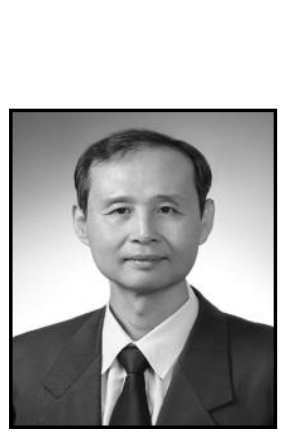

\section{Author}

Yong-Geol Shim, He received the B.S., M.S. and Ph.D. degrees in electronics engineering from Seoul National University, Korea, in 1982, 1984 and 1993 respectively. Since March 1988, he has been at Dankook University, where he is currently a professor. His research interests are information theory and channel coding. 